\title{
One Health e-Surveillance for Early Detection of Gastrointestinal Disease Outbreaks
}

\author{
Fernando Sánchez-Vizcaíno*1, Barry Rowlingson², Alan D Radford', Alison Hale², \\ Emanuele Giorgi², Sarah J O’Brien ${ }^{3}$, Susan Dawson ${ }^{4}$, Rosalind M Gaskell', Philip H \\ Jones $^{1}$, Tarek Menacere', Peter-John M Noble ${ }^{4}$, Maya Wardeh' ${ }^{1}$ and Peter Diggle ${ }^{2}$
}

\author{
'University of Liverpool, Institute of Infection and Global Health, Leahurst Campus, Neston, United Kingdom; ${ }^{2}$ University of Lancaster, \\ Faculty of Health and Medicine, Furness College, Lancaster, United Kingdom; ${ }^{3}$ University of Liverpool, Institute of Infection and \\ Global Health, Waterhouse Building (Block F), Liverpool, United Kingdom; ${ }^{4}$ University of Liverpool, School of Veterinary Science, \\ Leahurst Campus, Neston, United Kingdom
}

\section{Objective}

To describe how a real-time surveillance system for early detection of gastrointestinal disease (GI) outbreaks in small animal and human health is being developed by collecting electronic health records (EHRs) from veterinary practitioners and a telephone-based 24-hour medical triage service in the UK.

\section{Introduction}

In human and animal health, conventional approaches to preventing and controlling GI have not reduced the overall disease burden. In order to understand and mitigate shared GI aetiologies between humans and animals it is necessary to develop One Health Surveillance approaches that integrate data-sources contributed to by human and veterinary healthcare. Such approach is described here.

\section{Methods}

Veterinary data were collected electronically in real-time by SAVSNET, the Small Animal Veterinary Surveillance Network, from 102 UK veterinary practices (total of 197 premises) using a compatible version of practice management software. The study sampling frame included all dog and cat EHRs recorded within the SAVSNET database between January 2014 and July 2015. Each record included the animal signalment (including species, breed, sex, age, etc.), clinical free text, owner's postcode, treatment, syndrome information and results from a short questionnaire administered to veterinary surgeons and appended after $\sim 25 \%$ of GI consultation. The owner's postcode data were used to link each animal against UK databases containing human and environmental factors such as Indices of Multiple Deprivation.

A Bayesian spatio-temporal mixed effects binary regression model was used to model the incidence of GI in dogs and cats as a proportion of all presentations. The model was fitted to data between 01/11/2014 and 15/11/2014 using a bespoke Markov chain Monte Carlo algorithm to generate samples from the predictive distribution of the underlying spatio-temporal incidence surface. These samples were then used to compute predictive probabilities for exceedance of policy-relevant relative risk thresholds; a high predictive probability at a particular time and place gives an early warning of a possible GI outbreak. To test the ability of the model to detect GI outbreaks, a data set it was created with a fictitious premise having an excessive number of fake GI cases. The synthetic data is based on a typical premise in the SAVSNET dataset to ensure it reflects the characteristics of the genuine data. This premise is placed $1.5 \mathrm{~km}$ east and $3.9 \mathrm{~km}$ south of the chosen genuine premise. The outbreak is defined throughout the fictitious premise as the eight days from Monday, 03/11/2014 to Monday, 10/11/2014 inclusive. We run the model using data from $01 / 11 / 2014$ to $15 / 11 / 2014$.

Electronic data relating to human GI has recently become available by extracting spatial and temporal information from phone calls made to the NHS111 telephone-based 24-hour medical triage service by patients seeking medical advice about acute GI symptoms. The methodology that was initially developed using small animal data, as described above, can be easily adapted to analyse these data.

\section{Results}

EHR were obtained from 491,193 consultations $(361,203$ dogs, 129,990 cats). GI comprised $4.59 \%$ of canine and $3.63 \%$ of feline consultations, respectively. The final model included as explanatory variables age, species, weekend indicator, a measure of deprivation, animal's breed classified as purebred or crossbred and longitude / latitude effects. Predictive probabilities for a relative risk threshold of 1.2 or more were all comfortably greater than 0.5 identifying the faked outbreak.

\section{Conclusions}

This is the first demonstration of the feasibility of real-time syndromic surveillance in UK small animal practices. In future work, we intend to adapt the model to early detection of human GI outbreaks, and to investigate the possible inter-dependence of spatio-temporal variations in GI risk between companion animals and people.

\section{Keywords}

One health surveillance; early detection; gastrointestinal disease; UK; SAVSNET

\section{Acknowledgments}

We are grateful to BSAVA and to WT/HICF-T5-354 project (Integrate) for their funding of this work, and also to the many practitioners and pets' owners without whom these data would not be accessible.

*Fernando Sánchez-Vizcaíno

E-mail: fsvb@liv.ac.uk 